\title{
Adaptive-Mode Peak-to-Average Power Ratio Reduction Algorithm for OFDM-based Cognitive Radio
}

\author{
Rakesh Rajbanshi Alexander M. Wyglinski Gary J. Minden \\ Information and Telecommunication Technology Center \\ The University of Kansas \\ Lawrence, Kansas 66045-7612 \\ Email: \{rajbansh, alexw, gminden $\} @$ ittc.ku.edu
}

\begin{abstract}
In this paper, we present a novel low complexity algorithm for reducing the peak-to-average power ratio (PAPR) occurring in OFDM-based cognitive radios. Although several PAPR reduction algorithms exist in the literature, they are often only effective for specific scenarios. Our proposed algorithm exploits the agility of cognitive radio technology to rapidly choose and employ the appropriate PAPR reduction approach from a set of approaches to achieve a large decrease in PAPR, given the current operating conditions. The results show that for a wide range of operating conditions, the proposed algorithm achieves a large decrease in PAPR, unlikely the PAPR results when only a single reduction approach is employed across the same wide range.
\end{abstract}

\section{INTRODUCTION}

As access to available spectrum is becoming increasingly difficult, several researchers proposed the concepts of spectrum pooling and the use of non-contiguous portions of spectrum for high data rate transmission [1]. Non-contiguous OFDM (NCOFDM) is a promising candidate for such a flexible spectrum pooling system, where the implementation achieves high data rates via collective usage of a large number of non-contiguous subcarrier bands. Thus, the NC-OFDM can avoid interfering with incumbent users respecting their rights to the spectrum, while still being able to transmit information.

In general, OFDM offers high spectral efficiency, robustness to channel fading, immunity to impulse interference, and the capability to handle frequency-selective fading without resorting to complex channel equalization schemes [2,3]. However, one disadvantage of OFDM is that it could exhibit a large envelope variation in the time domain, which is often characterized by a large peak-to-average power ratio or PAPR (refer to Section III for details). When high PAPR occurs, the digital-to-analog (D/A) converter and power amplifier of the transmitter would require a large dynamic range to avoid amplitude clipping, thus increasing both power consumption and component cost.

To reduce PAPR in OFDM transceivers, there are several solutions proposed in the literature that could be employed by the system. These solutions include the use of error control coding [4-8] and constellation shaping techniques [9-11]. With respect to the latter, one constellation parameter that could be adjusted on a per-subcarrier basis is the subcarrier phase information. However, most of these algorithms possess a high computational complexity, especially for a large number of subcarriers. Data randomization, such as interleaving, can be employed to disrupt the long correlation patterns of a frame, hence reducing the PAPR of an OFDM signal [12]. However, interleaving is effective only when the data frames have moderate PAPR values. It was proposed that interleaving can be combined with selective mapping (SLM) techniques or partial transmit sequences (PTS) techniques for achieving greater reduction in PAPR [12]. However, combining interleaving with these techniques will substantially increase their computational complexity.

In this paper, we propose a novel low complexity algorithm that adaptively chooses which PAPR reduction approach, or combination thereof, to employ based on the current operating conditions, such as instantaneous PAPR. The PAPR reduction approaches employed by the proposed algorithm in this work are: (1) interleaving, and (2) per-subcarrier phase adjustments. The proposed algorithm is then evaluated within the framework of cognitive radio transceiver employing $\mathrm{NC}$ OFDM. The rest of the paper is organized as follows: A brief overview of an NC-OFDM transceiver is presented in Section II. The definition of PAPR is presented in Section III. The proposed algorithm is presented in Section IV. The simulation results from this work are presented in Section V and several conclusions are made in Section VI.

\section{NC-OFDM TRANSCEIVER}

A general schematic of an NC-OFDM transceiver employing the proposed PAPR reduction algorithm is shown in Fig. 1. The basic principle of NC-OFDM is to split a high-speed data stream, $x(n)$, into $N$ slower data streams that are transmitted simultaneously over orthogonal subcarriers. For NC-OFDM, the active subcarriers need not be contiguous and are located in unoccupied spectrum bands ${ }^{1}$. Without loss of generality, these streams are modulated using M-ary phase shift keying

\footnotetext{
${ }^{1}$ The usable spectrum bands are determined by dynamic spectrum sensing and channel sounding [13-15].
} 


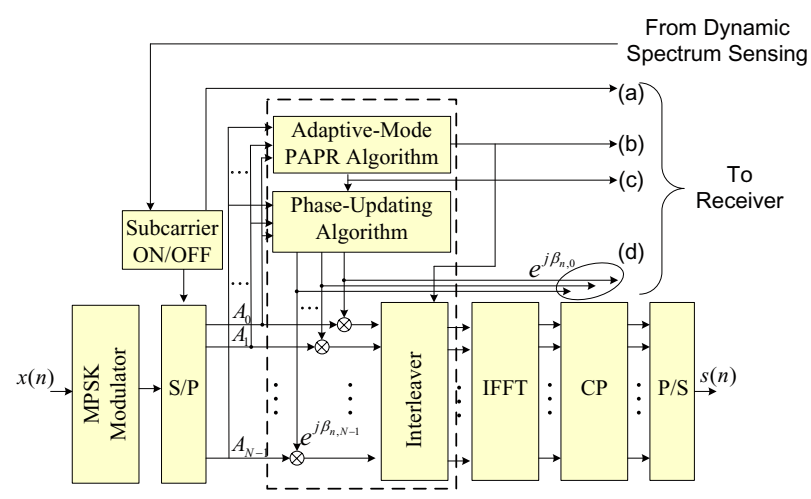

(a) Transmitter with proposed adaptive mode PAPR reduction algorithm.

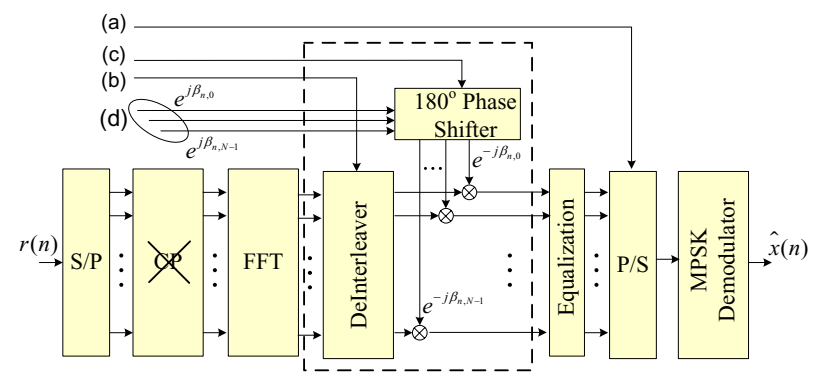

(b) Receiver with PAPR reduction information.

Fig. 1. Schematic of an NC-OFDM transceiver employing adaptive-mode PAPR reduction algorithm.

(MPSK). As shown in Fig. 1(a), the proposed adaptive-mode PAPR reduction algorithm decides upon computing phase adjustments, interleaving the data sequence, or a combination of both to reduce PAPR of an OFDM signal. The PAPR reduction information is also transmitted to the receiver. The inverse fast Fourier transform (IFFT) is then used to transform these modulated subcarrier signals into the time domain. Prior to transmission, a guard interval, with a length equal to or greater than the channel delay spread, is added to each OFDM symbol using the cyclic prefix $(\mathrm{CP})$ block in order to mitigate the effects of intersymbol interference (ISI). Following the parallel-to-serial $(\mathrm{P} / \mathrm{S})$ conversion, the baseband NC-OFDM signal, $s(n)$, is then passed through the transmitter radio frequency $(\mathrm{RF})$ chain, which amplifies the signal and upconverts it to the desired center frequency.

The complex envelope of a baseband NC-OFDM signal, consisting of all $N$ contiguous subcarriers over a time interval $[0, T]$, is given by

$$
s(t)=\frac{1}{N} \sum_{k=0}^{N-1} A_{k} e^{j 2 \pi k t / T}
$$

where $A_{k}=1 \angle \alpha_{k}=e^{j \alpha_{k}}$ is the symbol of the $k^{\text {th }}$ subcarrier $^{2}, T$ is the OFDM symbol duration, and $j=\sqrt{-1}$. The symbol of the $k^{\text {th }}$ unavailable subcarrier is $A_{k}=0$.

\footnotetext{
${ }^{2}$ For example, $\alpha_{k} \in\{0, \pi\}$ for BPSK signaling and $\alpha_{k} \in\left\{0, \frac{\pi}{2}, \pi, \frac{3 \pi}{2}\right\}$ for QPSK signaling.
}

The receiver downconverts the RF signal to baseband for processing, yielding the signal $r(n)$. Then, the signal is converted into parallel streams, the cyclic prefix is discarded, and the fast Fourier transform (FFT) is applied to transform the time domain data into the frequency domain. Distortions from the channel are equalized on per subcarrier basis. If the subcarrier phase information has been adjusted or the subcarriers have been interleaved, these modifications are reversed using the information from the transmitter. From the predetermined spectrum band, the subcarrier data is demodulated and converted into a reconstructed version of the original high-speed input, $\hat{x}(n)$.

\section{Peak-to-Average Power Ratio}

The PAPR of Eq. (1) is defined as the ratio between the maximum instantaneous power and the average power ${ }^{3}$, namely:

$$
\operatorname{PAPR}(s(t))=\frac{\max _{0 \leq t \leq T}|s(t)|^{2}}{E\left\{|s(t)|^{2}\right\}}
$$

where $E\{$.$\} denotes the expectation operator. The continuous$ time PAPR of $s(t)$ can be approximated using the discrete time PAPR, which is obtained using samples of the OFDM signal ${ }^{4}$. Therefore, in this paper, the discrete time PAPR will be employed in the analysis.

\section{Proposed PAPR Reduction Algorithm}

Although there has been much research conducted in the area of PAPR reduction, most of these solutions are suited for specific cases, depending on the similarity between the subcarrier information. Thus, a PAPR reduction approach that is effective for one scenario may not be suitable for another scenario. However, the proposed algorithm can choose the PAPR reduction approach best suited for a particular situation.

The objective of the proposed PAPR reduction algorithm is to choose the PAPR reduction mode that is capable of achieving a substantial decrease in PAPR. For instance, interleaving techniques can substantially decrease the PAPR, when the PAPR is moderate [12]. This is due to the fact that interleaving can be used to randomize the input sequence and disrupt the long correlation pattern of the input sequence. On the other hand, for very high PAPR values, phase-adjusting algorithms have shown to be the best choice for PAPR reduction [11, $18,19]$. This follows from the fact that binary or polyphase sequences with large out-of-phase aperiodic correlation values can result in signals with large PAPR values ${ }^{5}$. Thus, the resulting PAPR could be very high when the input sequence is highly biased. Although the probability of highly biased data frames is low over a long period of time, it is possible to have scenarios where data is biased for relatively short periods

\footnotetext{
${ }^{3}$ Without loss of generality, we can safely neglect the cyclic extension from the analysis since it does not contribute to the PAPR problem.

${ }^{4}$ It has been shown that an oversampling factor of four is sufficient to estimate the continuous PAPR of a BPSK system $[16,17]$.

${ }^{5}$ It follows from the fact that low out-of-phase aperiodic correlation values of binary or polyphase sequences results in a small value of the PAPR of the signal $[20,21]$.
} 


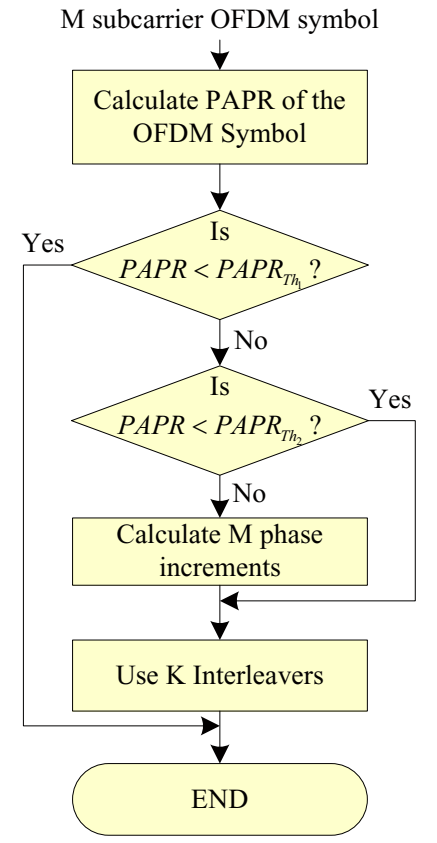

Fig. 2. Adaptive mode PAPR reduction algorithm.

of time ${ }^{6}$. Thus, the proposed adaptive mode PAPR reduction algorithm chooses the mode best suited for different transmission conditions in order to achieve a substantial reduction in PAPR for most scenarios.

Referring to the flow diagram of Fig. 2 and the transceiver schematic of Fig. 1(a), the proposed algorithm commences by computing the instantaneous PAPR of an OFDM signal, without any phase updating or interleaving, using the values of $A_{k}$. If the resulting PAPR value is less than a predefined threshold PAPR $\mathrm{Th}_{1}$, then the signal can be transmitted without any modification and the algorithm ends, i.e., neither the phase-updating algorithm block or interleaver block in Fig. 1(a) are employed. However, if the PAPR value is above the set threshold $\mathrm{PAPR}_{\mathrm{Th}_{1}}$, the algorithm must choose a PAPR reduction algorithm and lower this value. If the PAPR value is less than a set threshold $\mathrm{PAPR}_{\mathrm{Th}_{2}}$, the proposed algorithm choose the "interleaver" mode, i.e., the interleaver block in Fig. 1(a), where an interleaver is applied to the data sequence. Since the algorithm has $K$ interleavers available, the interleaver yielding the greatest decrease in PAPR is chosen. If the instantaneous PAPR is greater than $\mathrm{PAPR}_{\mathrm{Th}_{2}}$, the algorithm enters the "phase adjustment" mode, i.e., the phase-updating block of Fig. 1(a), where the subcarrier phases are adjusted to achieve a reduction in PAPR. If the PAPR reduction is insufficient, the algorithm will also employ one of the $K$ interleavers to the phase-updated subcarriers to achieve a larger PAPR reduction.

\footnotetext{
${ }^{6}$ For example, transmitting uncoded image data from a webcam, when the room is dimly lit, can result in data being highly biased.
}

\section{Simulation Results}

\section{A. Simulation Setup}

We now consider an NC-OFDM transceiver employing 512 BPSK-modulated subcarriers with $0 \%$ of the subcarriers being deactivated ${ }^{7}$. The percentage of subcarriers transmitting the same BPSK symbol, e.g., "1", varies from $50 \%$, i.e., unbiased scenario, to $99 \%$, i.e., highly biased scenario. For each scenario, 100, 000 BPSK-modulated NC-OFDM symbols were evaluated and the resulting PAPR values averaged. When generating the complementary cumulative density function (CCDF) plots of the PAPR for an NC-OFDM transceiver, 100,000 BPSK-modulated NC-OFDM symbols were employed for a given PAPR value. Finally, results for the two modes of operation of the proposed adaptive mode PAPR reduction algorithm are shown: (1) interleavers-only mode, and (2) phase adjustment with interleavers ${ }^{8}$.

\section{B. PAPR Results}

The mean and standard deviation of the PAPR for an NC-OFDM transceiver, employing only the best of the $K$ interleavers, are plotted in Fig. 3. We observe that when the percentage of subcarriers employing the same BPSK symbol is around $50 \%$, the interleaver approach appears to limit the PAPR to about $8 \mathrm{~dB}$. However, as the percentage increases beyond $60 \%$, the effectiveness of the interleavers to reduce the PAPR diminishes. Also notice how as the number of available interleavers increases, the PAPR can be reduced by as much as $0.5 \mathrm{~dB}$ for $K=16$ interleavers.

In Fig. 4, the mean and standard deviation of the PAPR for an NC-OFDM signal employing a phase updating algorithm and four interleavers are shown. We observe that with the combination of the phase updating algorithm and the interleavers, the mean PAPR does not exceed $7.5 \mathrm{~dB}$ when the percentage of subcarriers employing the same BPSK symbol ranges from $50 \%$ to $100 \%$. However, we know from other simulation results that the phase updating approach is ineffective at low percentages. Thus, to save computational and hardware complexity, the proposed algorithm can choose an interleaver-only mode when the percentage of identical subcarrier transmissions is below $60 \%$, and a combined mode when it is above $60 \%$.

To reduce the amount of overhead information required to perform phase adjustment PAPR reduction, the phase updates can be grouped together to reduce the information overhead to the receiver. In this case, as the subcarrier group size, $N_{\text {grp }}$, increases in the number of subcarriers, the PAPR reduction slightly degrades due to the loss in flexibility. Finally, notice how for percentages less than $60 \%$ that the PAPR values are the same as those in Fig. 3. This implies that at percentages around $50 \%$, phase adjustments do not have any significant

\footnotetext{
${ }^{7}$ With all the subcarriers are active, this would yield the worst case PAPR scenario.

${ }^{8}$ In this work, we employ the random interleaver approach used by Jayalath and Tellambura [12], and the phase updating approach of Rajbanshi, Wyglinski, and Minden [22], which is based on several similar approaches [18, 19].
} 


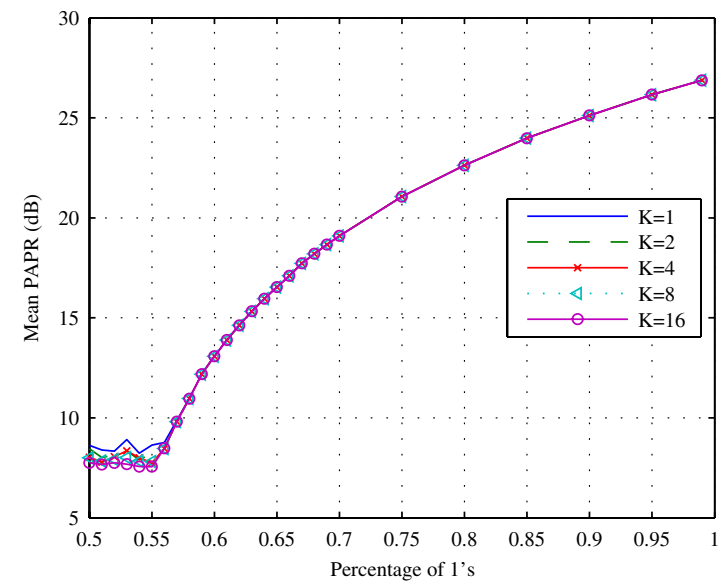

(a) $\mathrm{N}=512$

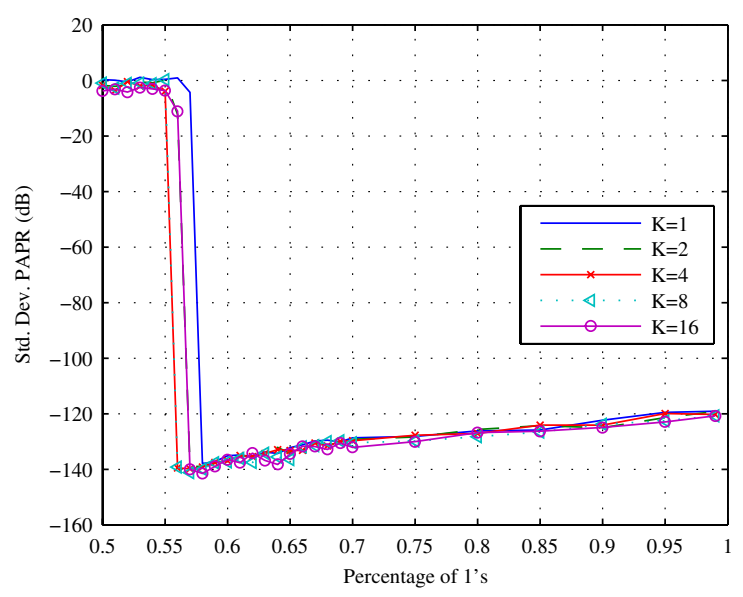

(b) $\mathrm{N}=512$

Fig. 3. PAPR statistics for BPSK modulated NC-OFDM signals with $N=$ 512 subcarriers and $K$ interleavers

impact on the reduction of the PAPR. Therefore, the proposed adaptive mode PAPR reduction algorithm would reduce the computational complexity by choosing only interleavers in this case.

The CCDF of the PAPR for an NC-OFDM transmission is plotted in Fig. 5, where the only PAPR technique being employed by the transceiver is only $K=4$ interleavers. It is evident from these results that the PAPR statistics improve with an increasing number of available random interleavers, relative to the case when no PAPR reduction is performed. Moreover, combining the phase adjustment approach and $K=$ 4 interleavers results in even better PAPR performance, relative to only employing interleavers. However, as the subcarrier group size, $N_{\mathrm{grp}}$, increases in the number of subcarriers, the PAPR reduction performance of the combined approach begins to deteriorate. Fig. 6 shows the PAPR CCDF when the number of available interleaver varies from $K=4$ to $K=16$. The observed trend is that as $K$ increases, the PAPR performance of the transceiver improves, with the probability that the PAPR which exceeds $8.6 \mathrm{~dB}$ is less than $0.1 \%$ when $K=16$

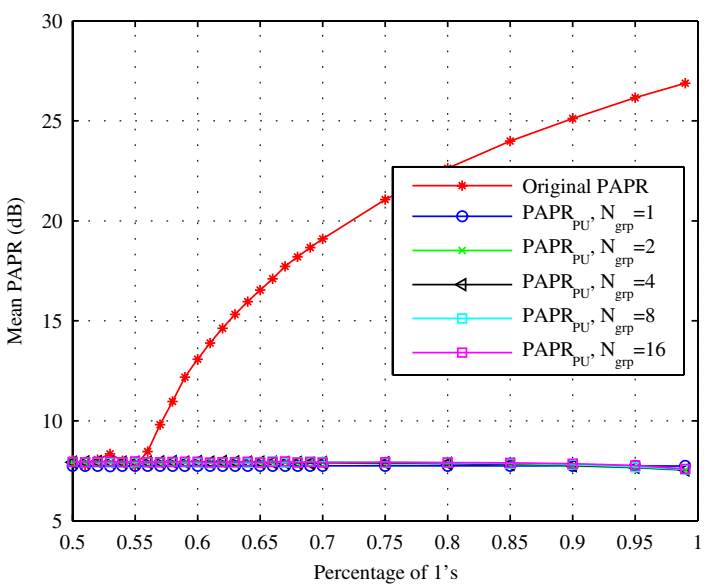

(a) $\mathrm{N}=512$

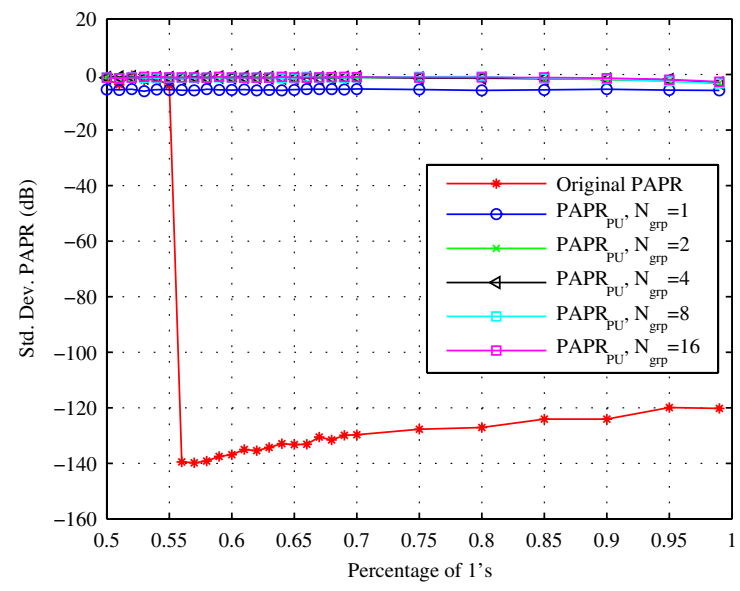

(b) $\mathrm{N}=512$

Fig. 4. PAPR statistics for BPSK modulated NC-OFDM signals with $N=$ 512 subcarriers and $K=4$ interleavers

interleavers.

\section{CONCLUSION}

In this paper, we presented a novel algorithm for the reduction of PAPR. The algorithm adaptively selects the appropriate approach to achieve the largest possible decrease in PAPR, given the available PAPR reduction approaches. In this work, we employed two PAPR reduction approaches: (1) interleaving, and (2) subcarrier phase adjustment. The results show when the approaches are employed across a wide range of operating conditions, they only achieve substantial performance gains for a small subset of cases. However, "gearshifting" between approaches achieves much better results across a wider range of scenarios.

\section{ACKNOWLEDGEMENTS}

The financial support provided by the National Science Foundation (NSF) through grants ANI-0230786 and ANI0335272 is gratefully acknowledged. 


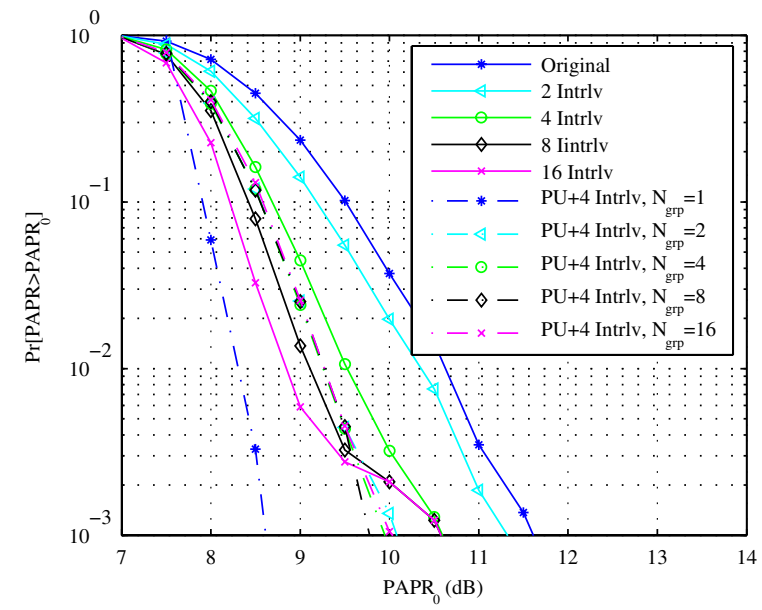

Fig. 5. CCDF of BPSK NC-OFDM signal PAPR with phase updating and random interleavers

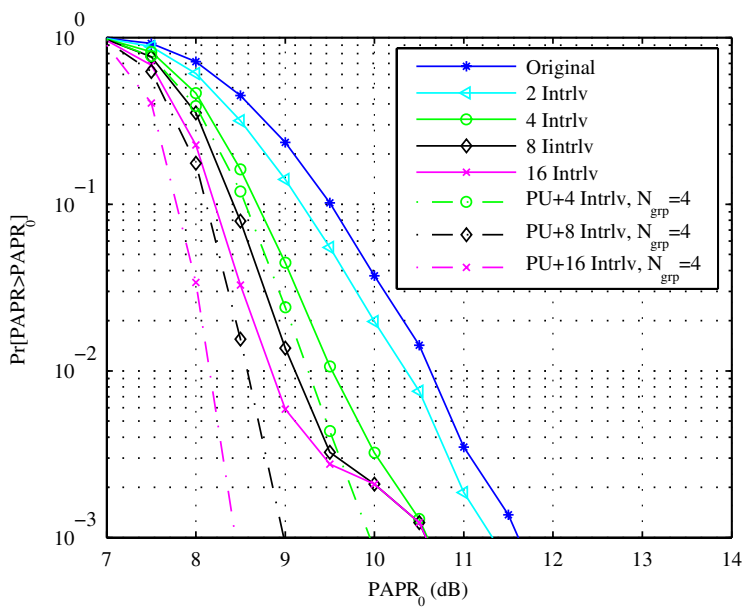

Fig. 6. CCDF of BPSK NC-OFDM signal PAPR with phase updating and random interleavers

\section{REFERENCES}

[1] T. A. Weiss and F. K. Jondral, "Spectrum pooling: an innovative strategy for the enhancement of spectrum efficiency," IEEE Commun. Mag, vol. 43, pp. S8 - 14, Mar. 2004.

[2] J. A. C. Bingham, "Multicarrier modulation for data transmission: an idea whose time has come," IEEE Commun. Mag, vol. 28, pp. 5-14, May 1990.

[3] B. R. Saltzberg, "Comparison of single-carrier and multitone digital modulation for ADSL applications," IEEE Commun. Mag, vol. 36, pp. 114-121, Nov. 1998.

[4] H. Ahn, Y. M. Shin, and S. Im, "A block coding scheme for peak to average power ratio reduction in an orthogonal frequency division multiplexing system," in Proc. 51st IEEE Veh. Technol. Conf. - Spring, vol. 1, (Tokyo, Japan), pp. 56 - 60, May 2000.

[5] C. Tellambura, "A coding technique for reducing peak to average power ratio in OFDM," in Proc. IEEE Global Telecommun. Conf., vol. 5 , (Sydney, Australia), pp. 2783 - 2787, Nov. 1998.

[6] T. A. Wilkison and A. E. Jones, "Minimization of the peak to mean envelope power ratio of multicarrier transmission schemes by block coding," in Proc. 45th IEEE Veh. Technol. Conf., vol. 2, (Chicago, IL, USA), pp. 825 - 829, July 1995.

[7] Y. Zhang, A. Yongacoglu, J. Chouinard, and L. Zhang, "OFDM peak power reduction by subblock coding and its extended versions," in Proc. 49th IEEE Veh. Technol. Conf. - Spring, vol. 1, (Houston, TX, USA), pp. 695 - 699, May 1999.

[8] R. Van Nee, "OFDM codes for peak to average power reduction and error correction," in Proc. IEEE Global Telecommun. Conf., vol. 1, (London, UK), pp. 740 - 744, Nov. 1996.

[9] R. W. Bauml, R. F. H. Fischer, and J. B. Huber, "Reducing the peak to average power ratio of multicarrier modulation by selective mapping," Electron. Lett., vol. 32, pp. 2056 - 2057, Oct. 1996.

[10] S. H. Muller and J. B. Huber, "OFDM with reduced peak to average power ratio by optimum combination of partial transmit sequences," Electron. Lett., vol. 33, pp. 368 - 369, Feb. 1997.

[11] H. Nikookar and K. S. Lidsheim, "Random phase updating algorithm for OFDM transmission with low PAPR," IEEE Trans. Broadcast, vol. 48, pp. 123 - 128, June 2002.

[12] A. D. S. Jayalath and C. Tellambura, "The use of interleaving to reduce the peak to average power ratio of an OFDM signal," in Proc. IEEE Global Telecommun. Conf., vol. 1, (San Francisco, CA, USA), pp. 82 86, Nov. 2000.

[13] F. Weidling, D. Datla, V. Petty, P. Krishnan, and G. J. Minden, "A framework for RF spectrum measurements and analysis," in Proc. IEEE Int. Symp. New Frontiers Dynamic Spectr. Access Networks, vol. 1, (Baltimore, MD, USA), pp. 573-576, Nov. 2005.

[14] S. Shankar, C. Cordeiro, and K. Challapali, "Spectrum agile radios: Utilization and sensing architectures," in Proc. IEEE Int. Symp. New Frontiers Dynamic Spectr. Access Networks, vol. 1, (Baltimore, MD, USA), pp. 160-169, Nov. 2005.

[15] Defence Advanced Research Projects Agency (DARPA), "The next generation (XG) program.” http://www.darpa.mil/ato/programs/XG/.

[16] C. Tellambura, "Computation of the continuous time PAR of an OFDM signal with BPSK subcarriers," IEEE Commun. Lett., vol. 5, pp. 185187, May 2001.

[17] H. Yu and G. Wei, "Computation of the continuous time PAR of an OFDM signal," in Proc. IEEE Int. Conf. Acoust., Speech, Signal Process., vol. 2003, (Hong Kong, China), pp. IV - 529-31, Apr. 2003.

[18] D. R. Gimlin and C. R. Patisaul, "On minimizing the peak-to-average power ratio for the sum of $\mathrm{N}$ sinusoids," IEEE Trans. Commun., vol. 41, pp. 631-635, Apr. 1993.

[19] S. Narahashi and T. Nojima, "New phasing scheme of N-multiple carriers for reducing peak-to-average power ratio," Electron. Lett., vol. 30, pp. 1382-1383, Aug. 1994.

[20] C. Tellambura, "Upper bound on peak factor of N-multiple carriers," Electron. Lett., vol. 33, pp. 1608-1609, Sept. 1997.

[21] N. Y. Ermolova and P. Vainikainen, "On the relationship between peak factor of a multicarrier signal and aperiodic autocorrelation of the generating sequence," IEEE Commun. Lett., vol. 7, pp. 107-108, Mar. 2003.

[22] R. Rajbanshi, A. Wyglinski, and G. Minden, "Phase adjusting OFDM transceivers for peak to average power ratio reduction." To be submitted. 